\title{
Connectivity Analysis in Vehicular Networks with Slight Traffic Interference
}

\author{
Bin Pan $\left.{ }^{1}\right)^{1}$ and Hao Wu $\mathbb{D D}^{1,2,3}$ \\ ${ }^{1}$ State Key Laboratory of Rail Traffic Control and Safety, Beijing Jiaotong University, Beijing 100044, China \\ ${ }^{2}$ Beijing Key Laboratory of Intelligent Traffic Data Safety and Privacy Protection Technology, Beijing Jiaotong University, \\ Beijing 100044, China \\ ${ }^{3}$ Beijing Engineering Research Center of High-Speed Railway Broad-Band Mobile Communications, Beijing Jiaotong University, \\ Beijing 100044, China \\ Correspondence should be addressed to Bin Pan; binpan@bjtu.edu.cn
}

Received 28 August 2018; Accepted 25 November 2018; Published 5 December 2018

Academic Editor: Petros Nicopolitidis

Copyright (C) 2018 Bin Pan and Hao Wu. This is an open access article distributed under the Creative Commons Attribution License, which permits unrestricted use, distribution, and reproduction in any medium, provided the original work is properly cited.

\begin{abstract}
This paper analyzes the network connectivity in vehicular networks with slight traffic interference, i.e., small-scale traffic accident. In this paper, we develop an analytical model for highway scenarios or sparse urban scenarios, where the slight traffic interference can make the vehicles slow down rather than block the traffic flow. When a traffic accident occurs, it is necessary to inform the nearby vehicles to slow down in order to reduce congestion at the accident location. Once they pass by, they can return to the normal value. Consequently, we can divide an entire road into two or three subsections, which helps us to analyze the connectivity performance. In addition, we analyze the impact of several key parameters on connectivity probability, including vehicle arrival rate, vehicle communication range, length of road, vehicle normal speed, and safe speed. All analytical results are verified through Monte Carlo simulation experiments. The simulation results are very close to analytical results, which means that the analytical results are accurate and the analytical model we propose is effective.
\end{abstract}

\section{Introduction}

Vehicular Ad Hoc Networks (VANETs), as special Mobile Ad Hoc Networks (MANETs), have attracted much attention in recent years. For future Intelligent Transportation System (ITS), VANETs can promise various applications in enhancing the road safety, improving traffic efficiency and providing entertainment services [1]. In VANETs, network connectivity for vehicles is one of the most significant performance $[2,3]$. Many researches have focused on the network connectivity in both highway and urban scenarios. However, most previous studies considered the normal road conditions. In practice, something unexpected may happen on the road, such as traffic accident or road maintenance. We consider smallscale traffic accidents and road maintenance as slight traffic interference, which can only make the vehicles slow down, rather than block the traffic flow. When a traffic interference occurs, it is necessary to inform the vehicles which will pass by the interference location to slow down. Once they pass by the interference location, their speeds can return to the normal value. Therefore, slight traffic interference could affect the connectivity performance of vehicles driving on the road. Connectivity analysis considering the slight traffic interference is useful to design the routing protocols and vehicular network architecture in special circumstances.

In this paper, we focus on the network connectivity on unidirectional road segment with a small-scale traffic accident. In particular, the road segment can be in a highway scenario or a sparse urban scenario, which means that the traffic accident can make the vehicles slow down, rather than block the traffic flow. The same as previous studies, the important metric we focus on is the connectivity probability. Here, network connectivity can be defined that if any two vehicles moving on the road segment can be connected through onehop or multihop Vehicle-to-Vehicle (V2V) communication, the whole network can be considered connected. For calculating the steady-state connectivity probability, we derived a connectivity analytical model, taking into consideration the 
traffic accident. In our considered road scenario, the location of traffic accident is uniformly distributed on the road. When a traffic accident (e.g., vehicle collision) occurred, the vehicle that collided with others could inform the vehicles behind within the communication range to slow down. Therefore, the traffic accident could divide the entire road segment into two or three segments with different vehicle speeds. In sparse VANETs scenario, the vehicle arrival follows Poisson process [4]. In the analytical model, there are several key parameters that have an influence on the connectivity performance, such as vehicle arrival rate, vehicle communication range, the length of entire road, vehicle normal speed, and safe speed. We verified the effectiveness of our analytical model through simulation experiments and discussed the impact of different parameters on the connectivity performance.

The main contributions of this paper are as follows:

(i) We developed an analytical model to analyze the connectivity probability of vehicles in a sparse road scenario with a traffic accident.

(ii) Based on proposed analytical model, we analyzed and discussed the impact of different key parameters on the connectivity.

(iii) All analytical results can be verified through Monte Carlo simulation method in MATLAB. The simulation results are very close to analytical results, which means that the analytical results are accurate and our proposed analytical model is effective.

The remainder of this paper is organized as follows. Section 2 reviews the related works about network connectivity in both highway and city scenario. Section 3 describes the system model and analyzes the network connectivity in detail. Section 4 verifies the analytical results through Monte Carlo simulation experiments and analyzes the impact of several key parameters on network connectivity. Section 5 summarizes the whole paper and proposes research prospects for future work.

\section{Related Works}

There are many studies focused on the connectivity in highway scenarios recently. In [5], Zhang et al. proposed a mathematical model to analyze both multihop uplink and downlink connectivity for infrastructure-based vehicular networks, where uplink connectivity probability indicates the user satisfaction level and downlink connectivity probability represents service coverage performance of a vehicular network. Simulation results reveal the impact of several parameters on the two performance metrics. In [6], Yousefi et al. investigated the connectivity using $M / G / \infty$ queuing model in high speed highways. Adding some mobile nodes with larger communication range, connectivity performance can be much improved. In addition, they also studied the case with fixed Roadside Units (RSUs). In [7], based on a mearsured case study, Cheng et al. analyzed the impact of intervehicle spacing distributions on connectivity in highway scenario. The intervehicle spacing with light traffic density follows the exponential distribution, while in the scenario with high traffic density, the Generalized Extreme Value (GEV) distribution is more suitable to model the intervehicle spacing compared with exponential distribution. In [8], Shao et al. investigated the connectivity performance in the free-flow state and proposed a connectivity-aware routing protocol. They analyzed the connectivity probability in both $\mathrm{V} 2 \mathrm{~V}$ and Vehicle-to-Infrastructure (V2I) communication scenarios, respectively. The relationship between connectivity probability and system throughput can be derived based on a Markov analytical model. In order to improve the system throughput, Shao et al. designed a multichannel reservation scheme taking into account network connectivity and variable traffic status. In [9], Yousefi et al. proposed an analytical model to study connectivity in vehicular highway networks. They utilized a $M / D / \infty$ queueing model to obtain the explicit form of the expected connectivity distance. The impact of different systems parameters (such as vehicle communication range, vehicle speed distribution, and traffic flow density) on the connectivity can be also obtained through the analytical model. In [10], Wu et al. investigated the connectivity properties in a mobile linear network considering high mobility of nodes and strict delay constraint. They proposed a new mobility model and a novel geometry-assisted analytical method to indicate the node distribution in steady state and to study the statistical properties of network connectivity. The conclusions can apply to delay constrained and tolerant networks.

For city block scenario, researchers also paid much attention. In [11], Viriyasitavat et al. designed a comprehensive framework to study the connectivity performance in urban vehicular networks. This framework can derive some closed-form results which help to understand the relationship between network connectivity and critical system parameters (i.e., traffic density, communication range, size of a road block and traffic light mechanisms). In [12], Zhang et al. derived a closed-form polynomial expression of the connectivity probability in two-dimensional lattice networks by using a recursive decomposition approach. Then, the proposed approach can be applied to a realistic urban scenario to obtain the directed connectivity. In [13], Hoque et al. studied multihop connectivity in urban scenario on the basis of the real trajectory of taxi. A novel algorithm was proposed to analyze the connectivity property and network partitioning. Using the efficient storage technique and computation mechanism, the space-time complexity can be reduced obviously. In [14], Naboulsi et al. studied the instantaneous topology of realistic urban scenarios, i.e., Cologne and Zurich. The results showed that a poor connectivity could cause limited availability and reliability. The analysis also indicated the unrealistic connected topologies can be caused by simplistic mobility models.

Under certain conditions, the vehicle density is different in different sections of one road. In [15], Khabazian et al. developed an analytical model that nodes can arrive at the highway or depart from the highway through the entry/exit points. They investigated the probability distribution of a node's location and node population sizes. Moreover, the statistical properties for connectivity can be also derived, such as mean cluster size and the probability that nodes can 
form a single cluster. In [16], Zheng et al. paid attention to the connectivity problem for highway road with one entry and exit. They developed an analytical model to derive the connectivity probability considering several critical system parameters, such as vehicle arrival rate, vehicle speed, and the probability that vehicles drive through the entry and exit point. The two scenarios that no RSU deployed and one RSU deployed are both investigated.

\section{System Model and Analysis of Connectivity}

In this section, we first describe the network scenario and system model. Then we give a detailed analysis for connectivity probability of vehicles driving on unidirectional road segment with a traffic accident.

3.1. System Model. As shown in Figure 1, we consider a multilane unidirectional road, which is denoted by $[0, d]$, where $d$ is the length of the entire road. The accident location $z$ is uniformly distributed on the entire road, i.e., $z \in[0, d]$. As mentioned above, the road segment can be in a highway scenario or a sparse urban scenario, ensuring the accident can only make the vehicles behind within communication range $R_{v}$ slow down, rather than block the traffic flow. Therefore, the traffic accident could divide the entire road segment into two or three subsegments with different vehicle speeds. Vehicles in the normal segment keep the normal speed $v_{1}$, while driving in the deceleration segment slow down with the safe speed $v_{2}$, i.e., $v_{1}>v_{2}$. In this paper, we neglect the acceleration and deceleration process of vehicles at the critical point of two different segment (i.e., the transition time between normal speed $v_{1}$ and safe speed $v_{2}$ ). For instance, the acceleration of regular vehicles is usually greater than $6.5 \mathrm{~m} / \mathrm{s}^{2}$, which indicates the speed transition with a span of $10 \mathrm{~m} / \mathrm{s}$ could be achieved within $1.5 \mathrm{~s}$. The transition time is far shorter than the sojourn time that the vehicles stay in road segment. The vehicles arriving at the road entry follow a Poisson process with arrival rate $\lambda$. Hence, in normal segment $\left[0, z-R_{v}\right]$ and $[z, d]$, the number of vehicles per meter follows the Poisson distribution with $\rho_{1}=\lambda / v_{1}$, while $\rho_{2}=\lambda / v_{2}$ in deceleration segment $\left[z-R_{v}, z\right]$. follows:

In this paper, we define the network connectivity as

(i) The two vehicles are considered connected, if their intervehicle spacing is not larger than the communication range of vehicles.

(ii) The subsegment is considered connected, if any two adjacent vehicles on this subsegment are connected.

(iii) The two adjacent subsegments are considered connected, if there are at least two connected vehicles which are, respectively, located on this two subsegments.

(iv) The entire road is considered connected, if any two adjacent subsegments in this road are connected.

3.2. Analysis of Connectivity. According to different traffic accident location $z \in[0, d]$, the network connectivity can

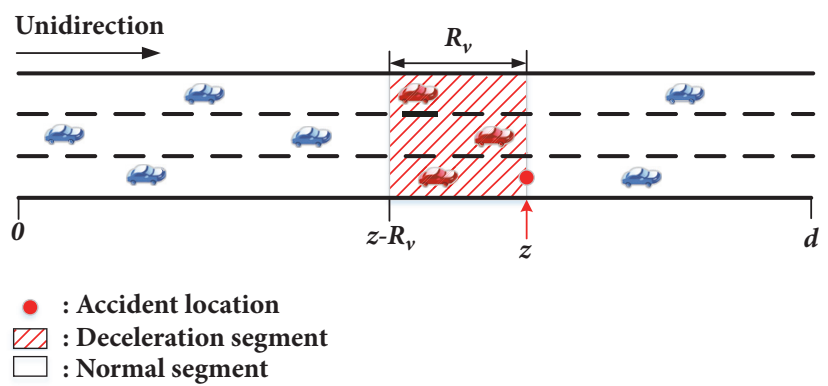

FIGURE 1: Network scenario.

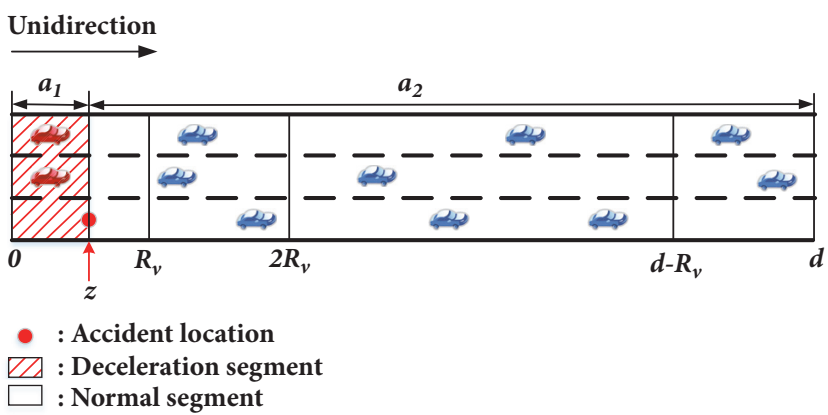

Figure 2: The situation of Case 1: $z \in\left[0, R_{v}\right]$.

be analyzed in the four cases below (under the assumption $\left.d>3 R_{v}\right)$ :

(i) Case 1: The traffic accident location $z \in\left[0, R_{v}\right]$.

(ii) Case 2: The traffic accident location $z \in\left[R_{v}, 2 R_{v}\right]$.

(iii) Case 3: The traffic accident location $z \in\left[2 R_{v}, d-R_{v}\right]$.

(iv) Case 4: The traffic accident location $z \in\left[d-R_{v}, d\right]$.

Case $1\left(z \in\left[0, R_{v}\right]\right)$. In Case 1 , as shown in Figure 2, the road segment $[0, z]$ can be connected obviously, if there are vehicles driving on the road segment. Therefore, we only need to analyze the connectivity of road segment $[z, d]$ and the connectivity between $[0, z]$ and $[z, d]$. If there is no vehicle driving on the road segment $[0, z]$, we only need to calculate the connectivity probability of road segment $[z, d]$. For convenience of description, we divide the entire road segment $[0, d]$ into two sub-segments $a_{1}$ and $a_{2}$, denoting $[0, z]$ and $[z, d]$, respectively.

In order to facilitate analysis, define the main notations to be used. Let $\operatorname{Pr}\{X\}$ denote the probability that the event $X$ occurs. Let $H_{s}$ denote the event that there are vehicles on segment $s$ and $C_{s}$ denote the event that the segment $s$ is connected. Hence, the events that there is no vehicle on segment $s$ and the segment $s$ is disconnected can be expressed by $\bar{H}_{s}$ and $\bar{C}_{s}$, respectively. Let $C_{s_{1}, s_{2}}$ denote the event that the segment $s_{1}$ and $s_{2}$ are connected; i.e., there are at least two connected vehicles, which are, respectively, driving on segment $s_{1}$ and $s_{2}$. 
On the basis of the mathematical property of Poisson distribution, the probability that there are vehicles driving on segment $a_{1}$ can be calculated by

$$
\operatorname{Pr}\left\{H_{a_{1}}\right\}=1-\frac{\left(\rho_{2} \cdot z\right)^{0}}{0 !} \cdot e^{-\rho_{2} \cdot z}=1-e^{-\rho_{2} \cdot z} .
$$

Hence, the probability that there is no vehicle driving on segment $a_{1}$ can be expressed by

$$
\operatorname{Pr}\left\{\bar{H}_{a_{1}}\right\}=1-\operatorname{Pr}\left\{H_{a_{1}}\right\}=e^{-\rho_{2} \cdot z} .
$$

Similarly, the probabilities that there are vehicles driving on segment $a_{2}$ and there is no vehicle on segment $a_{2}$ are expressed by

$$
\operatorname{Pr}\left\{H_{a_{2}}\right\}=1-e^{-\rho_{1} \cdot(d-z)}
$$

and

$$
\operatorname{Pr}\left\{\bar{H}_{a_{2}}\right\}=e^{-\rho_{1} \cdot(d-z)}
$$

respectively.

Further, Case 1 can be divided into two subcases as follows:

(i) Case 1.1: There are vehicles driving on segment $a_{1}$.

(ii) Case 1.2: There is no vehicle driving on segment $a_{1}$.

Case 1.1. In Case 1.1, clearly, the vehicles driving on segment $a_{1}$ are connected, i.e.,

$$
\operatorname{Pr}\left\{C_{a_{1}}\right\}=1
$$

Therefore, the vehicles on segment $a_{2}$ have a great impact on network probability. Take into account the two subcases below:

(i) Case 1.1.1: There are vehicles driving on segment $a_{2}$.

(ii) Case 1.1.2: There is no vehicle driving on segment $a_{2}$.

In Case 1.1.1, we will calculate the connectivity probability $\operatorname{Pr}\left\{C_{a_{2}}\right\}$ of the segment $a_{2}$ and the connectivity probability $\operatorname{Pr}\left\{C_{a_{1}, a_{2}}\right\}$ between segment $a_{1}$ and segment $a_{2}$.

According to [17], the connectivity probability of one road segment with constant Poisson process can be given by

$$
\begin{aligned}
\operatorname{Pr}\left\{\lambda_{\rho}, R_{v}, s\right\}= & e^{-\lambda_{\rho} s} \sum_{j=0}^{\left\lfloor s / R_{v}\right\rfloor} \frac{(-1)^{j}}{j !}\left[\lambda_{\rho}\left(s-j R_{v}\right)\right]^{j-1} \\
& \times\left[j+\lambda_{\rho}(s-j R)\right] e^{\lambda_{\rho}\left(s-j R_{v}\right)}
\end{aligned}
$$

where $\lambda_{\rho}$ means the vehicle density (i.e., the number of vehicles per meter follows the Poisson process with rate $\lambda_{\rho}$ ), $R_{v}$ is the communication range of vehicles, $s$ is the length of the road segment, and $\lfloor x\rfloor$ is the operation to obtain the largest integer which is not greater than $x$. Hence, we can calculate

$$
\operatorname{Pr}\left\{C_{a_{2}}\right\}=\operatorname{Pr}\left\{\rho_{1}, R_{v}, d-z\right\}
$$

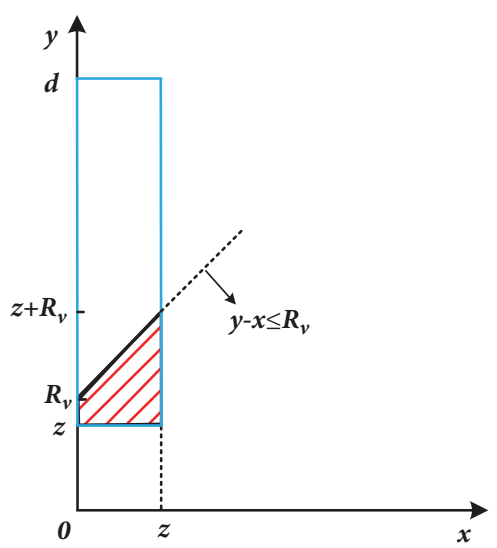

FIgURE 3: The diagram of calculating $p_{A B}$.

As for $\operatorname{Pr}\left\{C_{a_{1}, a_{2}}\right\}$, first of all, we calculate the connectivity probability that there are two connected vehicles, which are driving on segments $a_{1}$ and $a_{2}$, respectively. Assume that the vehicle $A$ is located at $L_{A}\left(0 \leq L_{A} \leq z\right)$, which is uniformly distributed on segment $a_{1}$, and the vehicle $B$ is located at $L_{B}$ $\left(z \leq L_{B} \leq d\right)$, which is uniformly distributed on segment $a_{2}$. Therefore, we can obtain the probability density function (PDF) of $L_{A}$ and $L_{B}$ below:

$$
\begin{aligned}
& f_{L_{A}}(x)=\frac{1}{z}, \quad 0 \leq x \leq z \\
& f_{L_{B}}(y)=\frac{1}{d-z}, \quad z \leq y \leq d
\end{aligned}
$$

Define $p_{A B}$ as the probability that the vehicles $A$ and $B$ are connected, i.e., the probability that the distance between vehicle $A$ and vehicle $B$ is not greater than communication range $R_{v}$. Hence, we have

$$
p_{A B}=\operatorname{Pr}\left\{y-x \leq R_{v}\right\}=\frac{2 R_{v}-z}{2(d-z)},
$$

which is equal to the ratio between the dashed area and the area of rectangle with blue border, as shown in Figure 3.

Then, we calculate the probability that there are at least two connected vehicles, which are driving on segments $a_{1}$ and $a_{2}$, respectively. On the basis of $p_{A B}$, the probability that a vehicle driving on segment $a_{2}$ cannot be connected with any of vehicles on segment $a_{1}$ can be expressed by

$$
\begin{aligned}
q_{a_{1}, a_{2}} & =\frac{1}{1-\operatorname{Pr}\left\{\bar{H}_{a_{1}}\right\}} \sum_{k=1}^{\infty}\left(1-p_{A B}\right)^{k} \operatorname{Pr}\left\{n_{a_{1}}=k\right\} \\
& =\frac{1}{e^{\rho_{2} z}-1}\left(e^{\left(2 d-2 R_{v}-z\right) / 2(d-z) \cdot \rho_{2} z}-1\right),
\end{aligned}
$$

where $\operatorname{Pr}\left\{n_{s}=k\right\}$ denote the probability that there are $k$ vehicle nodes driving on road segment $s$. Therefore, we 
can obtain the probability that the segments $a_{1}$ and $a_{2}$ are connected as follows:

$$
\begin{aligned}
\operatorname{Pr}\left\{C_{a_{1}, a_{2}}\right\} & =1-\sum_{k=0}^{\infty} q_{a_{1}, a_{2}}^{k} \cdot \operatorname{Pr}\left\{n_{a_{2}}=k\right\} \\
& =1-e^{-\rho_{1}(d-z)\left(1-q_{a_{1}, a_{2}}\right)} .
\end{aligned}
$$

Thus, according to (5), (7), and (12), the connectivity probability of the entire road segment $[0, d]$ in Case 1.1 .1 can be calculated by

$$
p_{111}=\operatorname{Pr}\left\{C_{a_{1}}\right\} \cdot \operatorname{Pr}\left\{C_{a_{2}}\right\} \cdot \operatorname{Pr}\left\{C_{a_{1}, a_{2}}\right\} .
$$

In Case 1.1.2, because there is no vehicle driving on segment $a_{2}$, the connectivity probability of the entire road segment $[0, d]$ in Case 1.1.2 can be expressed by

$$
p_{112}=\operatorname{Pr}\left\{C_{a_{1}}\right\}=1 \text {. }
$$

Considering the two subcases above, the connectivity probability of the entire road $[0, d]$ in Case 1.1 can be expressed by

$$
p_{11}=\operatorname{Pr}\left\{H_{a_{2}}\right\} \cdot P_{111}+\operatorname{Pr}\left\{\bar{H}_{a_{2}}\right\} \cdot P_{112}
$$

Case 1.2. In Case 1.2, we only need to calculate the connectivity probability of the segment $[z, d]$, i.e.,

$$
p_{12}=\operatorname{Pr}\left\{C_{a_{2}}\right\} \text {. }
$$

Considering the analysis mentioned above in Case 1, the connectivity probability of the entire road $[0, d]$ in Case 1 can be expressed by

$$
p_{1}=\operatorname{Pr}\left\{H_{a_{1}}\right\} \cdot p_{11}+\operatorname{Pr}\left\{\bar{H}_{a_{1}}\right\} \cdot p_{12} .
$$

Case $2\left(z \in\left[R_{v}, 2 R_{v}\right]\right)$. In Case 2, as shown in Figure 4, the entire road segment $[0, d]$ can be divided into three subsegments $b_{1}, b_{2}$, and $b_{3}$, denoting $\left[0, z-R_{v}\right],\left[z-R_{v}, z\right]$, and $[z, d]$, respectively.

Similar to Case 1, the following probabilities are easy to obtain:

$$
\begin{aligned}
& \operatorname{Pr}\left\{H_{b_{1}}\right\}=1-e^{-\rho_{1}\left(z-R_{v}\right)} \\
& \operatorname{Pr}\left\{H_{b_{2}}\right\}=1-e^{-\rho_{2} R_{v}} \\
& \operatorname{Pr}\left\{H_{b_{3}}\right\}=1-e^{-\rho_{1}(d-z)} \\
& \operatorname{Pr}\left\{\bar{H}_{b_{1}}\right\}=e^{-\rho_{1}\left(z-R_{v}\right)} \\
& \operatorname{Pr}\left\{\bar{H}_{b_{2}}\right\}=e^{-\rho_{2} R_{v}} \\
& \operatorname{Pr}\left\{\bar{H}_{b_{3}}\right\}=e^{-\rho_{1}(d-z)}
\end{aligned}
$$

According to whether there are vehicles driving on segment $b_{2}$, Case 2 can be divided into two subcases as follows:

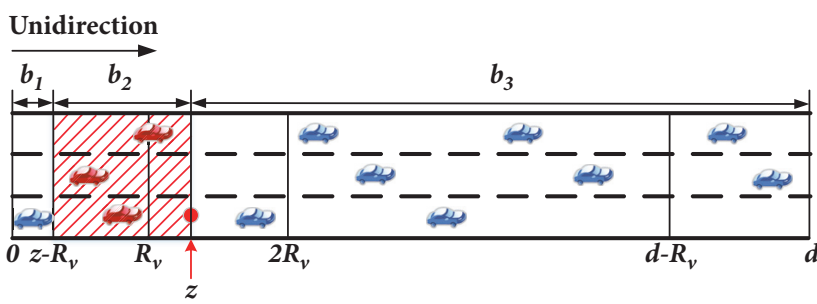

- : Accident location

ZA : Deceleration segment

: Normal segment

Figure 4: The situation of Case $2: z \in\left[R_{v}, 2 R_{v}\right]$.

(i) Case 2.1: There are vehicles driving on segment $b_{2}$.

(ii) Case 2.2: There is no vehicle driving on segment $b_{2}$.

In Case 2.1, clearly, the vehicles driving on segment $b_{2}$ are connected, i.e.,

$$
\operatorname{Pr}\left\{\mathrm{C}_{\mathrm{b}_{2}}\right\}=1 \text {. }
$$

Further, take in account the four subcases below:

(i) Case 2.1.1: There are vehicles driving on both segments $b_{1}$ and $b_{3}$.

(ii) Case 2.1.2: There are vehicles driving on segment $b_{1}$, but there is no vehicle driving on segment $b_{3}$.

(iii) Case 2.1.3: There is no vehicle driving on segment $b_{1}$, but there are vehicles driving on segment $b_{3}$.

(iv) Case 2.1.4: There is no vehicle driving on both segments $b_{1}$ and $b_{3}$.

In Case 2.1.1, it is obvious that the vehicles driving on segment $b_{1}$ are connected, i.e.,

$$
\operatorname{Pr}\left\{C_{b_{1}}\right\}=1 \text {. }
$$

Thus, we only need to analyze the connectivity probability $\operatorname{Pr}\left\{C_{b_{3}}\right\}, \operatorname{Pr}\left\{C_{b_{1}, b_{2}}\right\}$ and $\operatorname{Pr}\left\{C_{b_{2}, b_{3}}\right\}$. As for the connectivity probability $\operatorname{Pr}\left\{C_{b_{3}}\right\}$, it can be expressed by

$$
\operatorname{Pr}\left\{C_{b_{3}}\right\}=\operatorname{Pr}\left\{\rho_{1}, R_{v}, d-z\right\} .
$$

Using the same derivation method as $\operatorname{Pr}\left\{C_{a_{1}, a_{2}}\right\}$, the probability $\operatorname{Pr}\left\{C_{b_{1}, b_{2}}\right\}$ that road segments $b_{1}$ and $b_{2}$ are connected is given by

$$
\operatorname{Pr}\left\{C_{b_{1}, b_{2}}\right\}=1-e^{-\rho_{2} R_{v}\left(1-q_{b_{1}, b_{2}}\right)},
$$

where

$$
q_{b_{1}, b_{2}}=\frac{1}{e^{\rho_{1}\left(z-R_{v}\right)}-1}\left(e^{\left(z-R_{v}\right)^{2} / 2 R_{v} \cdot \rho_{1}}-1\right) .
$$

Similarly, the probability $\operatorname{Pr}\left\{C_{b_{2}, b_{3}}\right\}$ that segments $b_{2}$ and $b_{3}$ are connected is given by

$$
\operatorname{Pr}\left\{C_{b_{2}, b_{3}}\right\}=1-e^{-\rho_{1}(d-z)\left(1-q_{b_{2}, b_{3}}\right)},
$$


where

$$
q_{b_{2}, b_{3}}=\frac{1}{e^{\rho_{2} R_{v}}-1}\left(e^{\left(1-R_{v} / 2(d-z)\right) \cdot \rho_{2} R_{v}}-1\right) .
$$

Thus, the connectivity probability of the entire road segment $[0, d]$ in Case 2.1.1 can be expressed by

$$
\begin{aligned}
p_{211}= & \operatorname{Pr}\left\{C_{b_{1}}\right\} \cdot \operatorname{Pr}\left\{C_{b_{2}}\right\} \cdot \operatorname{Pr}\left\{C_{b_{3}}\right\} \cdot \operatorname{Pr}\left\{C_{b_{1}, b_{2}}\right\} \\
& \cdot \operatorname{Pr}\left\{C_{b_{2}, b_{3}}\right\} .
\end{aligned}
$$

In Case 2.1.2, there is no vehicle driving on segment $b_{3}$. Therefore, the connectivity probability of the entire road segment $[0, d]$ can be expressed by

$$
p_{212}=\operatorname{Pr}\left\{C_{b_{1}}\right\} \cdot \operatorname{Pr}\left\{C_{b_{2}}\right\} \cdot \operatorname{Pr}\left\{C_{b_{1}, b_{2}}\right\} .
$$

In Case 2.1.3, there is no vehicle driving on segment $b_{1}$. Therefore, the connectivity probability of the entire road segment $[0, d]$ can be expressed by

$$
p_{213}=\operatorname{Pr}\left\{C_{b_{2}}\right\} \cdot \operatorname{Pr}\left\{C_{b_{3}}\right\} \cdot \operatorname{Pr}\left\{C_{b_{2}, b_{3}}\right\} .
$$

In Case 2.1.4, there is no vehicle driving on segments $b_{1}$ and $b_{3}$. Therefore, the connectivity probability of the entire road segment $[0, d]$ can be expressed by

$$
p_{214}=\operatorname{Pr}\left\{C_{b_{2}}\right\}=1 \text {. }
$$

Regarding the four subcases above, the connectivity probability in Case 2.1 can be expressed by

$$
\begin{aligned}
p_{21}= & \operatorname{Pr}\left\{H_{b_{1}}\right\} \cdot \operatorname{Pr}\left\{H_{b_{3}}\right\} \cdot p_{211}+\operatorname{Pr}\left\{H_{b_{1}}\right\} \cdot \operatorname{Pr}\left\{\bar{H}_{b_{3}}\right\} \\
& \cdot p_{212}+\operatorname{Pr}\left\{\bar{H}_{b_{1}}\right\} \cdot \operatorname{Pr}\left\{H_{b_{3}}\right\} \cdot p_{213}+\operatorname{Pr}\left\{\bar{H}_{b_{1}}\right\} \\
& \cdot \operatorname{Pr}\left\{\bar{H}_{b_{3}}\right\} \cdot p_{214} .
\end{aligned}
$$

In Case 2.2, there is no vehicle driving on segment $b_{2}$. Only if one of the two following subcases occurs, it may be possible that the entire road segment $[0, d]$ is connected.

(i) Case 2.2.1: There are vehicles driving on segment $b_{1}$, but there is no vehicle driving on segment $b_{3}$.

(ii) Case 2.2.2: There is no vehicle driving on segment $b_{1}$, but there are vehicles driving on segment $b_{3}$.

In Case 2.2.1, the connectivity probability of the entire road segment $[0, d]$ can be calculated by

$$
p_{221}=\operatorname{Pr}\left\{C_{b_{1}}\right\}=1 \text {. }
$$

In Case 2.2.2, the connectivity probability of the entire road segment $[0, d]$ can be calculated by

$$
p_{222}=\operatorname{Pr}\left\{C_{b_{3}}\right\} \text {. }
$$

Regarding the two subcases above, the connectivity probability in Case 2.2 can be expressed by

$$
\begin{aligned}
p_{22}= & \operatorname{Pr}\left\{H_{b_{1}}\right\} \cdot \operatorname{Pr}\left\{\bar{H}_{b_{3}}\right\} \cdot p_{211}+\operatorname{Pr}\left\{\bar{H}_{b_{1}}\right\} \cdot \operatorname{Pr}\left\{H_{b_{3}}\right\} \\
& \cdot p_{222} .
\end{aligned}
$$

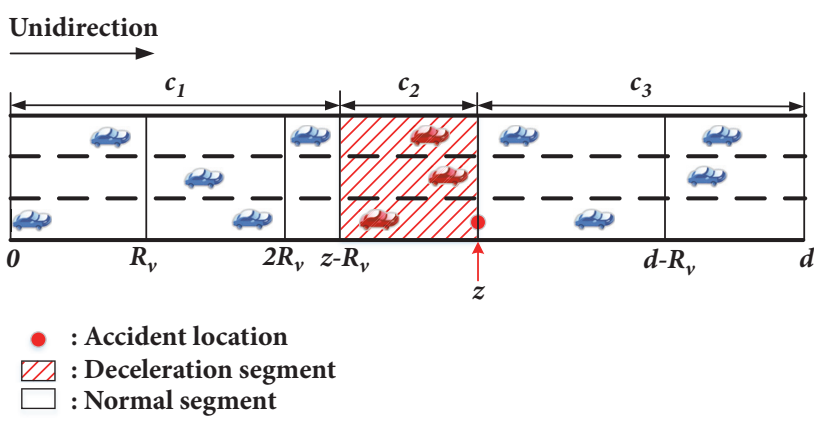

FIgURE 5: The situation of Case 3: $z \in\left[2 R_{v}, d-R_{v}\right]$.

Regarding all the analysis mentioned above in Case 2, the connectivity probability of the entire road segment $[0, d]$ in Case 2 is expressed by

$$
p_{2}=\operatorname{Pr}\left\{H_{b_{2}}\right\} \cdot p_{21}+\operatorname{Pr}\left\{\bar{H}_{b_{2}}\right\} \cdot p_{22} .
$$

Case $3\left(z \in\left[2 R_{v}, d-R_{v}\right]\right)$. In Case 3, as shown in Figure 5, the entire road segment $[0, d]$ can be divided into three subsegments $c_{1}, c_{2}$, and $c_{3}$, denoting $\left[0, z-R_{v}\right],\left[z-R_{v}, z\right]$, and $[z, d]$, respectively. The following probabilities are easy to obtain:

$$
\begin{aligned}
& \operatorname{Pr}\left\{H_{c_{1}}\right\}=1-e^{-\rho_{1}\left(z-R_{v}\right)} \\
& \operatorname{Pr}\left\{H_{c_{2}}\right\}=1-e^{-\rho_{2} R_{v}} \\
& \operatorname{Pr}\left\{H_{c_{3}}\right\}=1-e^{-\rho_{1}(d-z)} \\
& \operatorname{Pr}\left\{\bar{H}_{c_{1}}\right\}=e^{-\rho_{1}\left(z-R_{v}\right)} \\
& \operatorname{Pr}\left\{\bar{H}_{c_{2}}\right\}=e^{-\rho_{2} R_{v}} \\
& \operatorname{Pr}\left\{\bar{H}_{c_{3}}\right\}=e^{-\rho_{1}(d-z)}
\end{aligned}
$$

According to whether there are vehicles driving on segment $c_{2}$, Case 3 can be divided into two subcases as follows:

(i) Case 3.1: There are vehicles driving on segment $c_{2}$.

(ii) Case 3.2: There is no vehicle driving on segment $c_{2}$.

In Case 3.1, clearly, the vehicles driving on segment $c_{2}$ are connected, i.e.,

$$
\operatorname{Pr}\left\{C_{c_{2}}\right\}=1
$$

Further, take into account the four subcases below:

(i) Case 3.1.1: There are vehicles driving on both segments $c_{1}$ and $c_{3}$.

(ii) Case 3.1.2: There are vehicles driving on segment $c_{1}$, but there is no vehicle driving on segment $c_{3}$.

(iii) Case 3.1.3: There is no vehicle driving on segment $c_{1}$, but there are vehicles driving on segment $c_{3}$.

(iv) Case 3.1.4: There is no vehicle driving on both segments $c_{1}$ and $c_{3}$. 
In Case 3.1.1, we need to analyze the connectivity probabilities $\operatorname{Pr}\left\{C_{c_{1}}\right\}, \operatorname{Pr}\left\{C_{c_{3}}\right\}, \operatorname{Pr}\left\{C_{c_{2}, c_{1}}\right\}$, and $\operatorname{Pr}\left\{C_{c_{2}, c_{3}}\right\}$. As for connectivity probabilities $\operatorname{Pr}\left\{C_{c_{1}}\right\}$ and $\operatorname{Pr}\left\{C_{c_{3}}\right\}$, they can be expressed by

$$
\operatorname{Pr}\left\{C_{c_{1}}\right\}=\operatorname{Pr}\left\{\rho_{1}, R_{v}, z-R_{v}\right\}
$$

and

$$
\operatorname{Pr}\left\{C_{c_{3}}\right\}=\operatorname{Pr}\left\{\rho_{1}, R_{v}, d-z\right\}
$$

respectively.

Using the same derivation method as $\operatorname{Pr}\left\{C_{a_{1}, a_{2}}\right\}$, the probability $\operatorname{Pr}\left\{C_{c_{2}, c_{1}}\right\}$ that the segments $c_{2}$ and $c_{1}$ are connected can be given by

$$
\operatorname{Pr}\left\{C_{c_{2}, c_{1}}\right\}=1-e^{-\rho_{1}\left(z-R_{v}\right)\left(1-q_{c_{2}, c_{1}}\right)},
$$

where

$$
q_{c_{2}, c_{1}}=\frac{1}{e^{\rho_{2} R_{v}}-1}\left(e^{\left(1-R_{v} / 2\left(z-R_{v}\right)\right) \cdot \rho_{2} R_{v}}-1\right) .
$$

And the probability $\operatorname{Pr}\left\{C_{c_{2}, c_{3}}\right\}$ that the segments $c_{2}$ and $c_{3}$ are connected can be given by

$$
\operatorname{Pr}\left\{C_{c_{2}, c_{3}}\right\}=1-e^{-\rho_{1}(d-z)\left(1-q_{c_{2}, c_{3}}\right)}
$$

where

$$
q_{c_{2}, c_{3}}=\frac{1}{e^{\rho_{2} R_{v}}-1}\left(e^{\left(1-R_{v} / 2(d-z)\right) \cdot \rho_{2} R_{v}}-1\right) .
$$

Thus, the connectivity probability of the entire road segment $[0, d]$ in Case 3.1.1 can be expressed by

$$
\begin{aligned}
p_{311}= & \operatorname{Pr}\left\{C_{c_{1}}\right\} \cdot \operatorname{Pr}\left\{C_{c_{2}}\right\} \cdot \operatorname{Pr}\left\{C_{c_{3}}\right\} \cdot \operatorname{Pr}\left\{C_{c_{2}, c_{1}}\right\} \\
& \cdot \operatorname{Pr}\left\{C_{c_{2}, c_{3}}\right\} .
\end{aligned}
$$

In Case 3.1.2, there is no vehicle driving on segment $c_{3}$. Therefore, the connectivity probability of the entire road segment $[0, d]$ can be expressed by

$$
p_{312}=\operatorname{Pr}\left\{C_{c_{1}}\right\} \cdot \operatorname{Pr}\left\{C_{c_{2}}\right\} \cdot \operatorname{Pr}\left\{C_{c_{2}, c_{1}}\right\} .
$$

In Case 3.1.3, there is no vehicle driving on segment $c_{1}$. Therefore, the connectivity probability of the entire road segment $[0, d]$ can be expressed by

$$
p_{313}=\operatorname{Pr}\left\{C_{c_{2}}\right\} \cdot \operatorname{Pr}\left\{C_{c_{3}}\right\} \cdot \operatorname{Pr}\left\{C_{c_{2}, c_{3}}\right\} .
$$

In Case 3.1.4, there is no vehicle driving on segment $c_{1}$ and $c_{3}$. Therefore, the connectivity probability of the entire road segment $[0, d]$ can be expressed by

$$
p_{314}=\operatorname{Pr}\left\{C_{c_{2}}\right\}=1 .
$$

Regarding the four subcases above, the connectivity probability in Case 3.1 can be expressed by

$$
\begin{aligned}
p_{31}= & \operatorname{Pr}\left\{H_{c_{1}}\right\} \cdot \operatorname{Pr}\left\{H_{c_{3}}\right\} \cdot p_{311}+\operatorname{Pr}\left\{H_{c_{1}}\right\} \cdot \operatorname{Pr}\left\{\bar{H}_{c_{3}}\right\} \\
& \cdot p_{312}+\operatorname{Pr}\left\{\bar{H}_{c_{1}}\right\} \cdot \operatorname{Pr}\left\{H_{c_{3}}\right\} \cdot p_{313}+\operatorname{Pr}\left\{\bar{H}_{c_{1}}\right\} \\
& \cdot \operatorname{Pr}\left\{\bar{H}_{c_{3}}\right\} \cdot p_{314} .
\end{aligned}
$$

In Case 3.2, there is no vehicle driving on segment $c_{2}$. Only if one of the two following subcases occurs may it be possible that the entire road segment $[0, d]$ is connected:

(i) Case 3.2.1: There are vehicles driving on segment $c_{1}$, but there is no vehicle driving on segment $c_{3}$.

(ii) Case 3.2.2: There is no vehicle driving on segment $c_{1}$, but there are vehicles driving on segment $c_{3}$.

In Case 3.2.1, the connectivity probability of the entire road segment $[0, d]$ can be calculated by

$$
p_{321}=\operatorname{Pr}\left\{C_{c_{1}}\right\}
$$

In Case 3.2.2, the connectivity probability of the entire road segment $[0, d]$ can be calculated by

$$
p_{322}=\operatorname{Pr}\left\{C_{c_{3}}\right\} \text {. }
$$

Regarding the two subcases above, the connectivity probability in Case 3.2 can be expressed by

$$
\begin{aligned}
p_{32}= & \operatorname{Pr}\left\{H_{c_{1}}\right\} \cdot \operatorname{Pr}\left\{\bar{H}_{c_{3}}\right\} \cdot p_{321}+\operatorname{Pr}\left\{\bar{H}_{c_{1}}\right\} \cdot \operatorname{Pr}\left\{H_{c_{3}}\right\} \\
& \cdot p_{322} .
\end{aligned}
$$

Regarding all the analysis mentioned above in Case 3, the connectivity probability of the entire road segment $[0, d]$ in Case 3 is expressed by

$$
p_{3}=\operatorname{Pr}\left\{H_{c_{2}}\right\} \cdot p_{31}+\operatorname{Pr}\left\{\bar{H}_{c_{2}}\right\} \cdot p_{32} .
$$

Case $4\left(z \in\left[d-R_{v}, d\right]\right)$. In Case 4 , as shown in Figure 6, the entire road segment $[0, d]$ can be divided into three subsegments $d_{1}, d_{2}$, and $d_{3}$, denoting $\left[0, z-R_{v}\right],\left[z-R_{v}, z\right]$, and $[z, d]$, respectively.

Similar to Case 1 , the following probabilities are easy to obtain:

$$
\begin{aligned}
& \operatorname{Pr}\left\{H_{d_{1}}\right\}=1-e^{-\rho_{1}\left(z-R_{v}\right)} \\
& \operatorname{Pr}\left\{H_{d_{2}}\right\}=1-e^{-\rho_{2} R_{v}} \\
& \operatorname{Pr}\left\{H_{d_{3}}\right\}=1-e^{-\rho_{1}(d-z)} \\
& \operatorname{Pr}\left\{\bar{H}_{d_{1}}\right\}=e^{-\rho_{1}\left(z-R_{v}\right)} \\
& \operatorname{Pr}\left\{\bar{H}_{d_{2}}\right\}=e^{-\rho_{2} R_{v}} \\
& \operatorname{Pr}\left\{\bar{H}_{d_{3}}\right\}=e^{-\rho_{1}(d-z)}
\end{aligned}
$$

According to whether there are vehicles driving on segment $d_{2}$, Case 4 can be divided into two subcases as follows:

(i) Case 4.1: There are vehicles driving on segment $d_{2}$.

(ii) Case 4.2: There is no vehicle driving on segment $d_{2}$. 


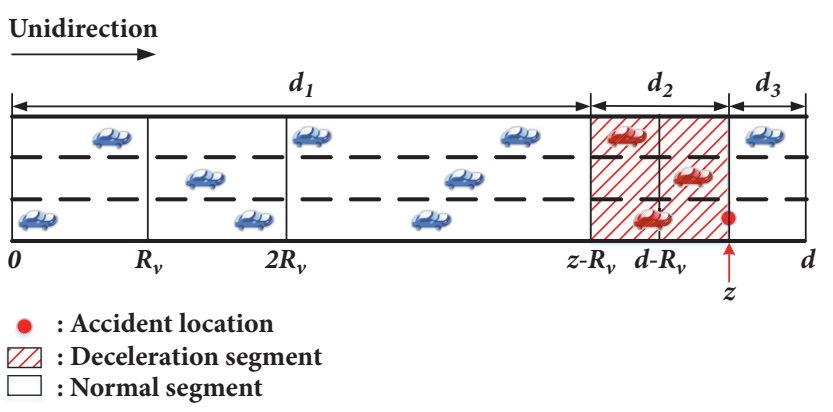

FIgURe 6: The situation of Case 4: $z \in\left[d-R_{v}, d\right]$.

In Case 4.1, clearly, the vehicles driving on segment $d_{2}$ are connected, i.e.,

$$
\operatorname{Pr}\left\{C_{d_{2}}\right\}=1
$$

Further, take into account the four subcases below:

(i) Case 4.1.1: There are vehicles driving on both segments $d_{1}$ and $d_{3}$.

(ii) Case 4.1.2: There are vehicles driving on segment $d_{1}$, but there is no vehicle driving on segment $d_{3}$.

(iii) Case 4.1.3: There is no vehicle driving on segment $d_{1}$, but there are vehicles driving on segment $d_{3}$.

(iv) Case 4.1.4: There is no vehicle driving on both segments $d_{1}$ and $d_{3}$.

In Case 4.1.1, it is obvious that the vehicles driving on segment $d_{3}$ are connected, i.e.,

$$
\operatorname{Pr}\left\{C_{d_{3}}\right\}=1
$$

Thus, we need to analyze the connectivity probabilities $\operatorname{Pr}\left\{C_{d_{1}}\right\}, \operatorname{Pr}\left\{C_{d_{2}, d_{1}}\right\}$, and $\operatorname{Pr}\left\{C_{d_{3}, d_{2}}\right\}$. As for the connectivity probability $\operatorname{Pr}\left\{C_{d_{1}}\right\}$, it can be expressed by

$$
\operatorname{Pr}\left\{C_{d_{1}}\right\}=\operatorname{Pr}\left\{\rho_{1}, R_{v}, z-R_{v}\right\} \text {. }
$$

Using the same derivation method as $\operatorname{Pr}\left\{C_{a_{1}, a_{2}}\right\}$, the probability $\operatorname{Pr}\left\{C_{d_{2}, d_{1}}\right\}$ that the segments $d_{2}$ and $d_{1}$ are connected can be driven by

$$
\operatorname{Pr}\left\{C_{d_{2}, d_{1}}\right\}=1-e^{-\rho_{1}\left(z-R_{v}\right)\left(1-q_{d_{2}, d_{1}}\right)},
$$

where

$$
q_{d_{2}, d_{1}}=\frac{1}{e^{\rho_{2} R_{v}}-1}\left(e^{\left(1-R_{v} / 2\left(z-R_{v}\right)\right) \cdot \rho_{2} R_{v}}-1\right) .
$$

And the probability $\operatorname{Pr}\left\{C_{d_{3}, d_{2}}\right\}$ that the segments $d_{3}$ and $d_{2}$ are connected can be driven by

$$
\operatorname{Pr}\left\{C_{d_{3}, d_{2}}\right\}=1-e^{-\rho_{2} R_{v}\left(1-q_{d_{3}, d_{2}}\right)},
$$

where

$$
q_{d_{3}, d_{2}}=\frac{1}{e^{\rho_{1}(d-z)}-1}\left(e^{(d-z)^{2} \rho_{1} / 2 R_{v}}-1\right) .
$$

Thus, the connectivity probability of the entire road segment $[0, d]$ in Case 4.1 .1 can be expressed by

$$
\begin{aligned}
p_{411}= & \operatorname{Pr}\left\{C_{d_{1}}\right\} \cdot \operatorname{Pr}\left\{C_{d_{2}}\right\} \cdot \operatorname{Pr}\left\{C_{d_{3}}\right\} \cdot \operatorname{Pr}\left\{C_{d_{2}, d_{1}}\right\} \\
& \cdot \operatorname{Pr}\left\{C_{d_{3}, d_{2}}\right\} .
\end{aligned}
$$

In Case 4.1.2, there is no vehicle driving on segment $d_{3}$. Therefore, the connectivity probability of the entire road segment $[0, d]$ can be expressed by

$$
p_{412}=\operatorname{Pr}\left\{C_{d_{1}}\right\} \cdot \operatorname{Pr}\left\{C_{d_{2}}\right\} \cdot \operatorname{Pr}\left\{C_{d_{2}, d_{1}}\right\} .
$$

In Case 4.1.3, there is no vehicle driving on segment $d_{1}$. Therefore, the connectivity probability of the entire road segment $[0, d]$ can be expressed by

$$
p_{413}=\operatorname{Pr}\left\{C_{d_{2}}\right\} \cdot \operatorname{Pr}\left\{C_{d_{3}}\right\} \cdot \operatorname{Pr}\left\{C_{d_{3}, d_{2}}\right\} .
$$

In Case 4.1.4, there is no vehicle driving on segments $d_{1}$ and $d_{3}$. Therefore, the connectivity probability of the entire road segment $[0, d]$ can be expressed by

$$
p_{414}=\operatorname{Pr}\left\{C_{d_{2}}\right\}=1 \text {. }
$$

Regarding the four subcases above, the connectivity probability in Case 3.1 can be expressed by

$$
\begin{aligned}
p_{41}= & \operatorname{Pr}\left\{H_{d_{1}}\right\} \cdot \operatorname{Pr}\left\{H_{d_{3}}\right\} \cdot p_{411}+\operatorname{Pr}\left\{H_{d_{1}}\right\} \\
& \cdot \operatorname{Pr}\left\{\bar{H}_{d_{3}}\right\} \cdot p_{412}+\operatorname{Pr}\left\{\bar{H}_{d_{1}}\right\} \cdot \operatorname{Pr}\left\{H_{d_{3}}\right\} \cdot p_{413} \\
& +\operatorname{Pr}\left\{\bar{H}_{d_{1}}\right\} \cdot \operatorname{Pr}\left\{\bar{H}_{d_{3}}\right\} \cdot p_{414} .
\end{aligned}
$$

In Case 4.2 , there is no vehicle driving on segment $d_{2}$. Only if one of the two following subcases occurs may it be possible that the entire road segment $[0, d]$ is connected:

(i) Case 4.2.1: There are vehicles driving on segment $d_{1}$, but there is no vehicle driving on segment $d_{3}$.

(ii) Case 4.2.2: There is no vehicle driving on segment $d_{1}$, but there are vehicles driving on segment $d_{3}$.

In Case 4.2.1, the connectivity probability of the entire road segment $[0, d]$ can be calculated by

$$
p_{421}=\operatorname{Pr}\left\{C_{d_{1}}\right\} \text {. }
$$

In Case 4.2 .2 , the connectivity probability of the entire road segment $[0, d]$ can be calculated by

$$
p_{422}=\operatorname{Pr}\left\{C_{d_{3}}\right\}=1 \text {. }
$$

Regarding the two subcases above, the connectivity probability in Case 3.2 can be expressed by

$$
\begin{aligned}
p_{42}= & \operatorname{Pr}\left\{H_{d_{1}}\right\} \cdot \operatorname{Pr}\left\{\bar{H}_{d_{3}}\right\} \cdot p_{411}+\operatorname{Pr}\left\{\bar{H}_{d_{1}}\right\} \\
& \cdot \operatorname{Pr}\left\{H_{d_{3}}\right\} \cdot p_{422} .
\end{aligned}
$$


Regarding all the analysis mentioned above in Case 4, the connectivity probability of the entire road segment $[0, d]$ in Case 4 is expressed by

$$
p_{4}=\operatorname{Pr}\left\{H_{d_{2}}\right\} \cdot p_{41}+\operatorname{Pr}\left\{\bar{H}_{d_{2}}\right\} \cdot p_{42} .
$$

Therefore, regarding all the analysis mentioned above, the connectivity probability of the entire road segment $[0, d]$ can be expressed by

$$
\begin{aligned}
p & =\frac{1}{d} \cdot\left(\int_{0}^{R_{v}} p_{1} d z+\int_{R_{v}}^{2 R_{v}} p_{2} d z+\int_{2 R_{v}}^{d-R_{v}} p_{3} d z\right. \\
& \left.+\int_{d-R_{v}}^{d} p_{4} d z\right) .
\end{aligned}
$$

\section{Simulation Results and Discussions}

In this section, we verify the accuracy and effectiveness of our proposed analytical model through simulation experiments. In addition, we analyze and discuss the impact of different key parameters on connectivity performance, including vehicle arrival rate $\lambda$, vehicle communication range $R_{v}$, the length of entire road $d$, vehicle normal speed $v_{1}$, and safe speed $v_{2}$. To perform the simulation experiments, we adopt Monte Carlo simulation method in MATLAB. For each simulation result, it is an average of $10^{5}$ trials. The simulation results are very close to analytical results, which means that the analytical results are accurate and our proposed analytical model is effective.

Figure 7 shows the impact of accident location on connectivity probability. From the curve in traffic accident condition, we can see that the connectivity probability increases first and then decreases with different accident location, which shows that the vehicles in the middle part of the road have a greater influence on the connectivity. When the traffic accident occurs at location 0 , the traffic flow in $[0, d]$ can be same as that in normal condition. Hence, the connectivity probability with accident location 0 is equal to that with normal condition. Note that the traffic accident, which makes the vehicles slow down rather than block the traffic flow, can improve the connectivity to a certain degree.

Figures 8-11 all show the impact of vehicle arrival rate on connectivity probability. Obviously, the connectivity probability increases with the increase of vehicle arrival rate. It is because that the vehicle density can increase with the increase of arrival rate, which improves the road connectivity. What is more, from Figures 8-11, we can see that the deviation between analytical result and simulation result decreases with the increase of vehicle arrival rate. The reason is that the vehicles have a relatively large arrival randomness and intervehicle spacing with a small arrival rate.

In addition, Figure 8 shows the impact of vehicle communication range on connectivity probability. The connectivity probability increases with the increase of vehicle communication range, which is because that the two vehicles can obtain a greater opportunity to be connected with a larger communication range. Note that the connectivity probability has a larger improvement with the communication range from $200 \mathrm{~m}$ to $300 \mathrm{~m}$, comparing with other communication

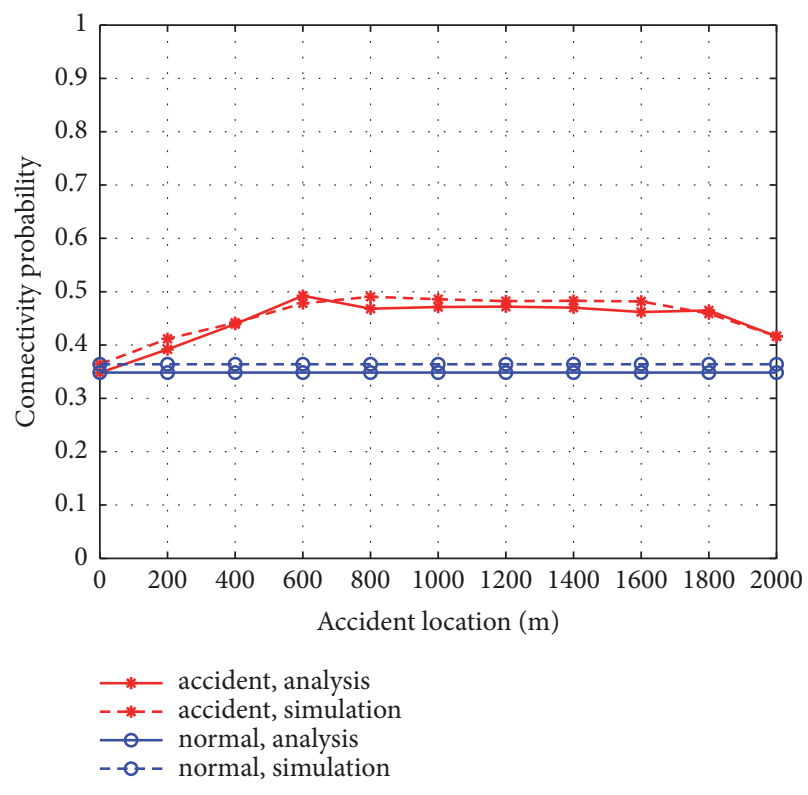

FIGURE 7: The impact of accident location on connectivity probability $\left(\lambda=0.2 \mathrm{veh} / \mathrm{s}, v_{1}=20 \mathrm{~m} / \mathrm{s}, v_{2}=10 \mathrm{~m} / \mathrm{s}, R_{v}=300 \mathrm{~m}\right.$, and $\left.d=2000 \mathrm{~m}\right)$.

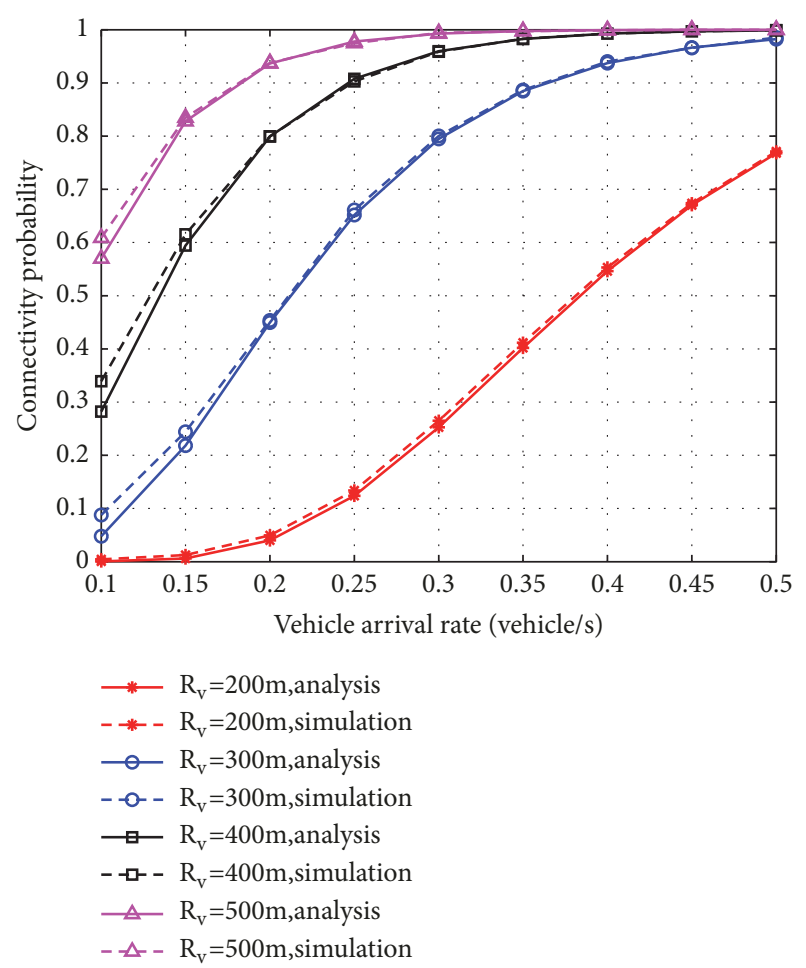

FIgURE 8: The impact of vehicle arrival rate and communication range on connectivity probability $\left(v_{1}=20 \mathrm{~m} / \mathrm{s}, v_{2}=10 \mathrm{~m} / \mathrm{s}\right.$, and $d=2000 \mathrm{~m})$.

range gaps. It can offer guidance to the design of signal transmitting power.

Figure 9 shows the impact of the length of entire road on connectivity probability. It can be seen that the connectivity probability decreases with the increase of the length of road. The reason is that, with constant probability that two adjacent 


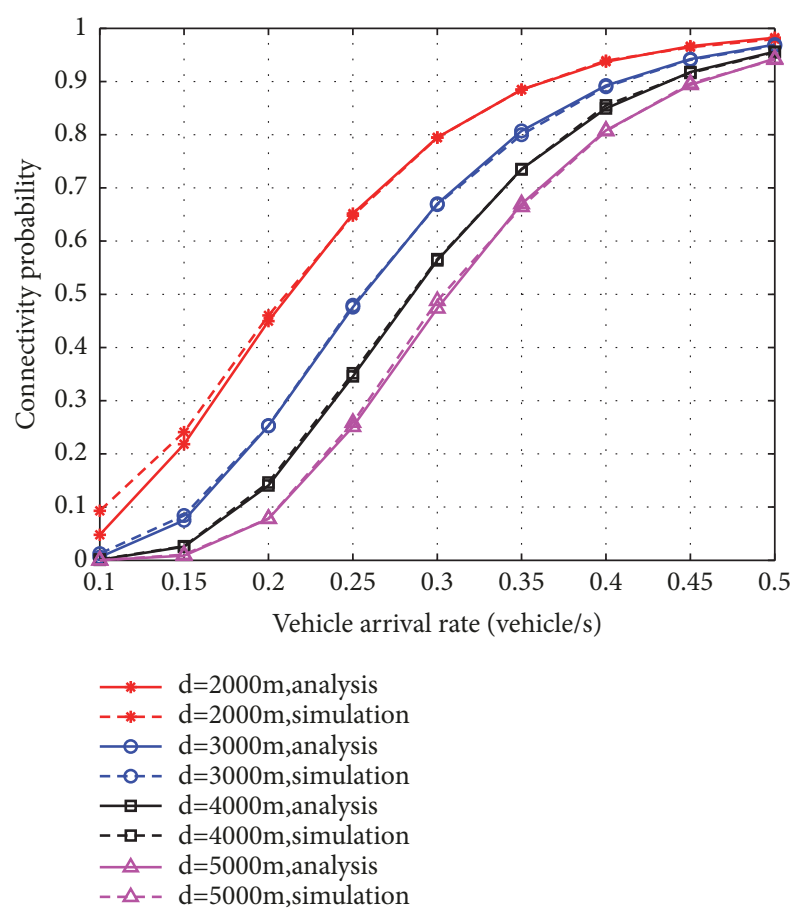

FIGURE 9: The impact of vehicle arrival rate and length of road on connectivity probability $\left(R_{v}=300 \mathrm{~m}, v_{1}=20 \mathrm{~m} / \mathrm{s}\right.$, and $\left.v_{2}=10 \mathrm{~m} / \mathrm{s}\right)$.

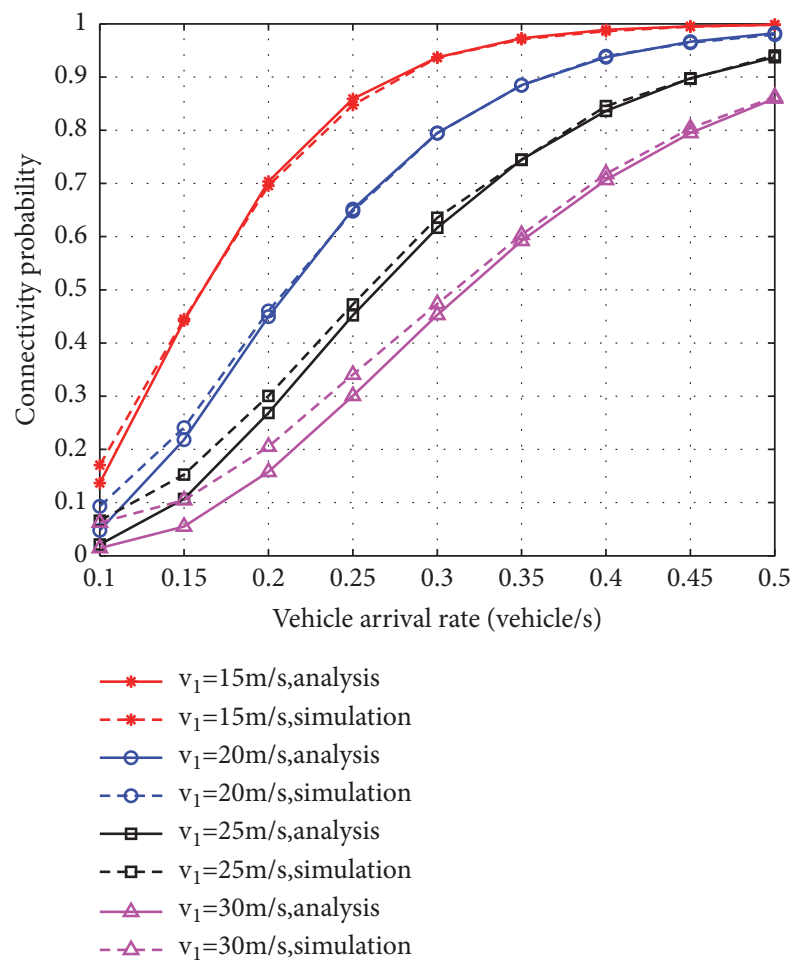

FIGURE 10: The impact of vehicle arrival rate and vehicle normal speed on connectivity probability $\left(R_{v}=300 \mathrm{~m}, v_{2}=10 \mathrm{~m} / \mathrm{s}, d=2000\right.$ $\mathrm{m})$.

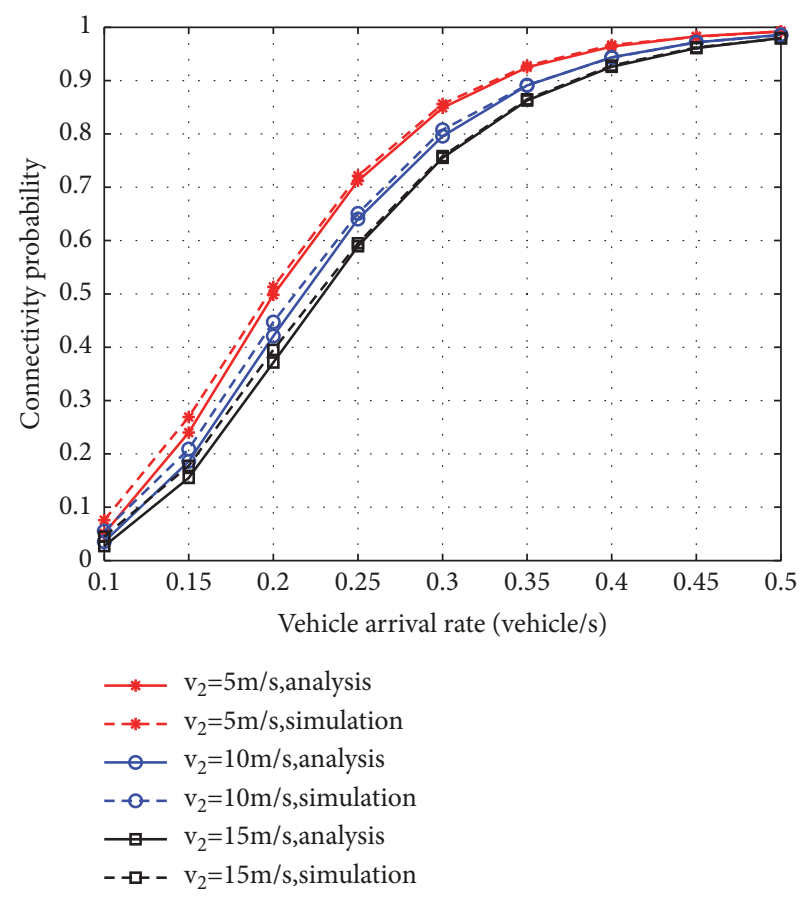

FIGURE 11: The impact of vehicle arrival rate and vehicle safe speed on connectivity probability $\left(R_{v}=300 \mathrm{~m}, v_{1}=20 \mathrm{~m} / \mathrm{s}\right.$, and $\left.d=2000 \mathrm{~m}\right)$.

vehicles are connected, the larger the length of road is, the larger the number of vehicles is, the smaller the connectivity probability of all vehicles is. Consequently, the connectivity probability decreases.

Figure 10 shows the impact of vehicle normal speed on connectivity probability. It can be seen that the connectivity probability decreases with the increase of vehicle normal speed. The reason is that the vehicle density in normal segment decreases with the increase of normal speed. Moreover, the normal segment occupies a relatively large proportion of the entire road. Therefore, the connectivity probability of the entire road decreases relatively obviously.

Figure 11 shows the impact of vehicle safe speed on connectivity probability. It can be seen that the connectivity probability decreases slightly with the increase of vehicle safe speed. The reason is the similar to the impact of normal speed on connectivity probability. However, the deceleration segment occupies a small proportion of the entire road, resulting in a slow decline in probability.

\section{Conclusions}

In this paper, we analyzed the connectivity in vehicular networks with slight traffic interference, i.e., small-scale traffic accident. An analytical model was proposed to calculate the connectivity probability of the entire road segment. The road segment can be in a highway scenario or a sparse urban scenario, which means that the slight traffic interference can only make the vehicles slow down, rather than block the traffic flow. When a traffic accident occurs, it is necessary to inform the vehicles within communication range which 
will pass by the accident location to slow down. Once passing by the accident location, their speeds can return to the normal value. Moreover, on the basis of the analytical model, we analyzed the impact of several key parameters on connectivity probability, including vehicle arrival rate, vehicle communication range, the length of road, vehicle normal speed, and safe speed. All analytical results are verified through simulation experiments. The simulation results are very close to analytical results, which means that the analytical results are accurate and our proposed analytical model is effective.

In the future, we will consider the acceleration and deceleration process in a more practical scenario, which is much more complicated. Note that we assume the vehicle arrival follows the Poisson process; namely, intervehicle spacing follows the exponential distribution. How about the network connectivity with other intervehicle spacing distribution? In addition, in order to facilitate the analysis, the length of deceleration segment is set to the value of vehicle communication range. The different cases with different values of the length of deceleration segment are also our future work.

\section{Data Availability}

The data used to support the findings of this study are available from the corresponding author upon request.

\section{Conflicts of Interest}

The authors declare that they have no conflicts of interest.

\section{Acknowledgments}

This paper is supported by the Fundamental Research Funds for the Central Universities under Grant nos. 2018JBZ102, 2017JBM312, and it is supported in part by NFSC Outstanding Youth under Grant no. 61725101.

\section{References}

[1] J. J. Blum, A. Eskandarian, and L. J. Huffman, "Challenges of intervehicle Ad Hoc networks," IEEE Transactions on Intelligent Transportation Systems, vol. 5, no. 4, pp. 347-351, 2004.

[2] K. Sampigethaya, M. Li, L. Huang, and R. Poovendran, "AMOEBA: robust location privacy scheme for VANET," IEEE Journal on Selected Areas in Communications, vol. 25, no. 8, pp. 1569-1589, 2007.

[3] D. Miorandi and E. Altman, "Connectivity in one-dimensional ad hoc networks: A queueing theoretical approach," Wireless Networks, vol. 12, no. 5, pp. 573-587, 2006.

[4] N. Wisitpongphan, F. Bai, P. Mudalige, V. Sadekar, and O. Tonguz, "Routing in sparse vehicular ad hoc wireless networks," IEEE Journal on Selected Areas in Communications, vol. 25, no. 8, pp. 1538-1556, 2007.

[5] W. Zhang, Y. Chen, Y. Yang et al., "Multi-hop connectivity probability in infrastructure-based vehicular networks," IEEE Journal on Selected Areas in Communications, vol. 300, no. 4, pp. 740-747, 2012.
[6] S. Yousefi, E. Altman, R. El-Azouzi, and M. Fathy, "Improving connectivity in vehicular ad hoc networks: An analytical study," Computer Communications, vol. 31, no. 9, pp. 1653-1659, 2008.

[7] L. Cheng and S. Panichpapiboon, "Effects of intervehicle spacing distributions on connectivity of VANET: a case study from measured highway traffic," IEEE Communications Magazine, vol. 50, no. 10, pp. 90-97, 2012.

[8] C. Shao, S. Leng, Y. Zhang, A. Vinel, and M. Jonsson, "Performance analysis of connectivity probability and connectivityaware MAC protocol design for platoon-based VANETs," IEEE Transactions on Vehicular Technology, vol. 64, no. 12, pp. 55965609, 2015.

[9] S. Yousefi, E. Altman, R. El-Azouzi, and M. Fathy, "Analytical model for connectivity in vehicular Ad Hoc networks," IEEE Transactions on Vehicular Technology, vol. 57, no. 6, pp. 33413356,2008

[10] J. Wu, "Connectivity of mobile linear networks with dynamic node population and delay constraint," IEEE Journal on Selected Areas in Communications, vol. 27, no. 7, pp. 1218-1225, 2009.

[11] W. Viriyasitavat, F. Bai, and O. K. Tonguz, "Dynamics of network connectivity in urban vehicular networks," IEEE Journal on Selected Areas in Communications, vol. 29, no. 3, pp. 515-533, 2011.

[12] L. Zhang, L. Cai, J. Pan, and F. Tong, "A new approach to the directed connectivity in two-dimensional lattice networks," IEEE Transactions on Mobile Computing, vol. 13, no. 11, pp. 2458-2472, 2014.

[13] M. A. Hoque, X. Hong, and B. Dixon, "Efficient multi-hop connectivity analysis in urban vehicular networks," Vehicular Communications, vol. 1, no. 2, pp. 78-90, 2014.

[14] D. Naboulsi and M. Fiore, "Characterizing the instantaneous connectivity of large-scale urban vehicular networks," IEEE Transactions on Mobile Computing, vol. 16, no. 5, pp. 1272-1286, 2017.

[15] M. Khabazian and M. K. M. Ali, "A performance modeling of connectivity in vehicular Ad Hoc networks," IEEE Transactions on Vehicular Technology, vol. 57, no. 4, pp. 2440-2450, 2008.

[16] J. Zheng and Y. Wang, "Connectivity analysis of vehicles moving on a highway with an entry and exit," IEEE Transactions on Vehicular Technology, no. 99, 1 page, 2018.

[17] S.-I. Sou and O. K. Tonguz, "Enhancing VANET connectivity through roadside units on highways," IEEE Transactions on Vehicular Technology, vol. 60, no. 8, pp. 3586-3602, 2011. 


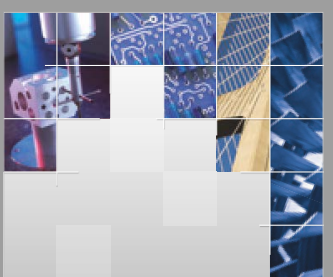

\section{Enfincering}
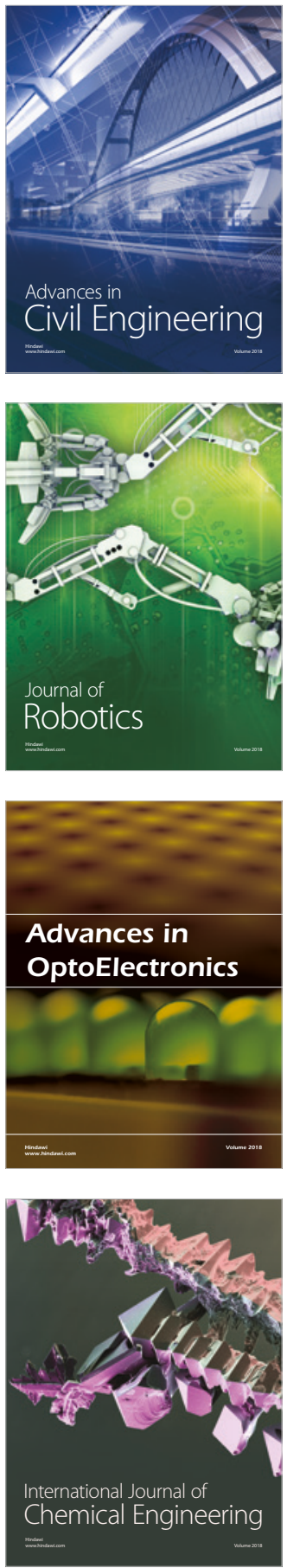

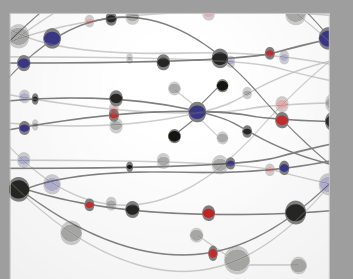

\section{Rotating \\ Machinery}

The Scientific World Journal

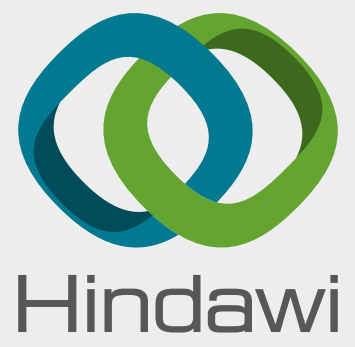

Submit your manuscripts at

www.hindawi.com
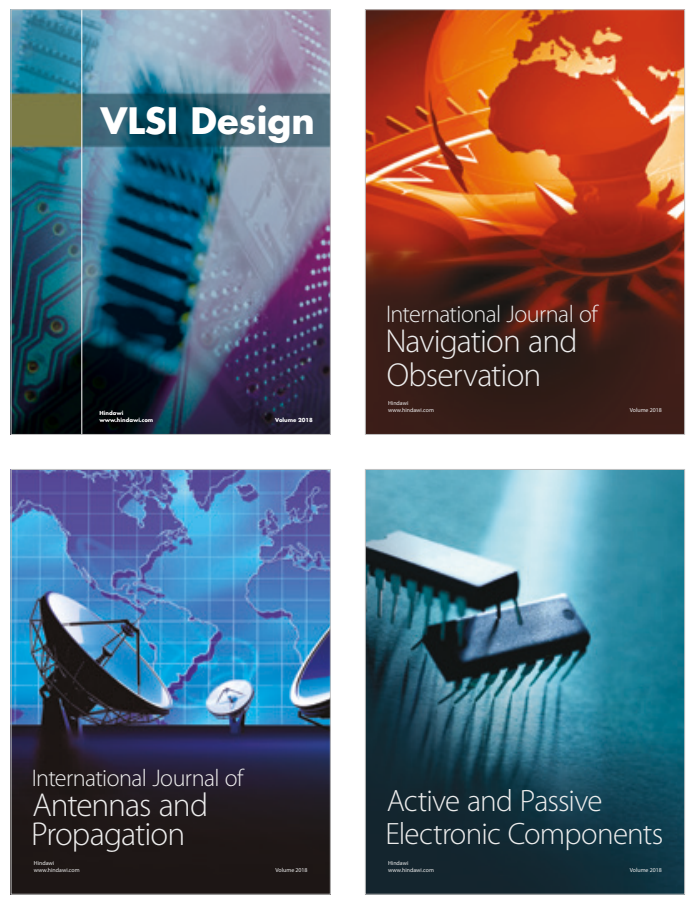
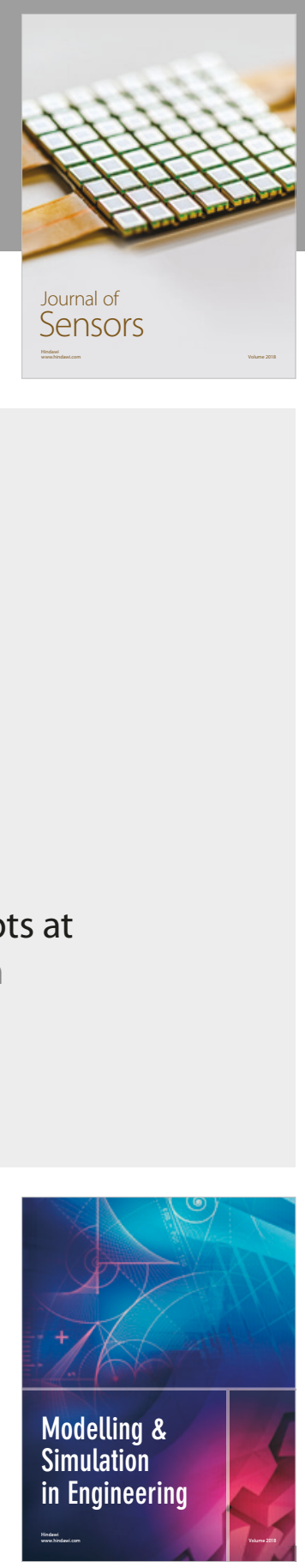

\section{Advances \\ Multimedia}
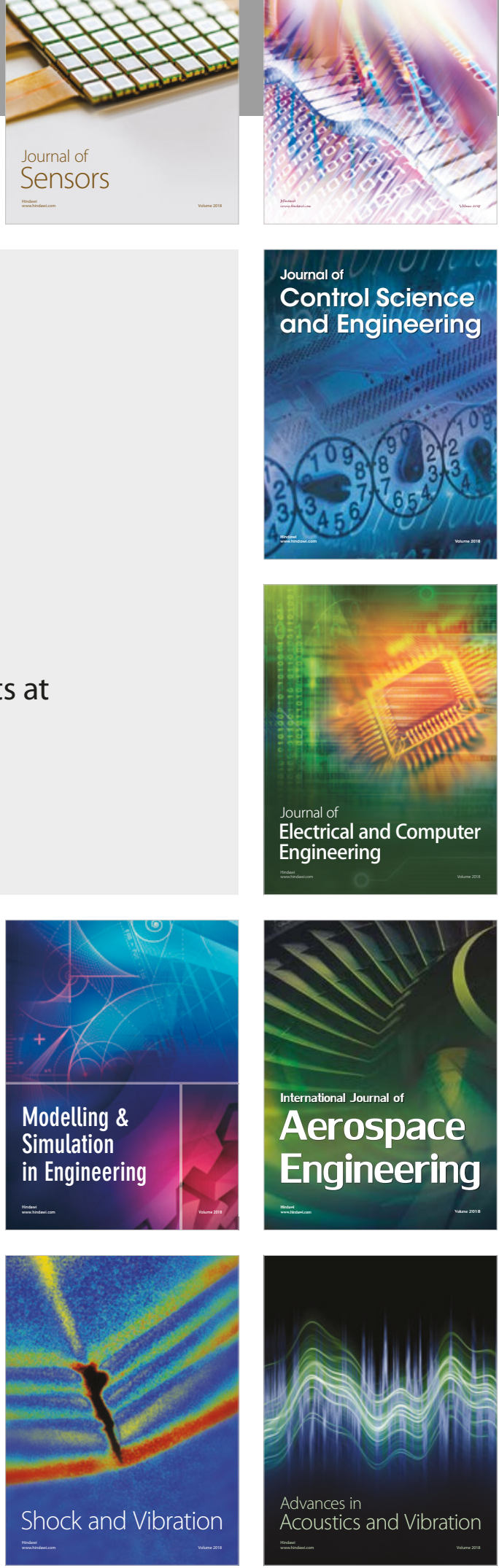